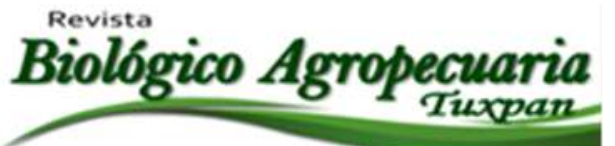

\title{
Secuestro de carbono en suelo cafetalero con alta pendiente en la Sierra de Santa Marta
}

\author{
Carbon sequestration in soil coffee with high slope in the Sierra de Santa Marta \\ Vázquez-Luna Dinora, Cuevas-Díaz María del Carmen, Perera-Escamilla Teresita de Jesús, Hernández Romero \\ Ángel Héctor ${ }^{1}$, Retureta Aponte Alejandro \\ Facultad de Ingeniería en Sistemas de Producción Agropecuaria. Universidad Veracruzana. \\ ${ }^{凶}$ Autor para correspondencia: aretureta@uv.mx
}

Recibido: $15 / 03 / 2018$
Aceptado: 10/05/2018

\section{RESUMEN}

En este estudio se analizó el potencial de secuestro de carbono de un suelo proveniente de un cafetal con sombra, influenciado por una pendiente del $34 \%$. El carbono total secuestrado a lo largo de la pendiente fue cuantificado mediante el aporte de la hojarasca, las ramas, el mantillo y el suelo. La población de bacterias fijadoras de nitrógeno de vida libre (BFNVL) y el contenido de materia orgánica (MO) fueron usados como indicadores de la calidad del suelo. Finalmente, se determinó el impacto de los beneficios económicos del secuestro de carbono bajo esas condiciones. Los resultados indicaron que el cafetal bajo sombra ofrece condiciones favorables para el secuestro de carbono, debido al aporte continuo de carbono proveniente de la hojarasca y las ramas, con la consecuente formación de mantillo; sin embargo, el secuestro de carbono total fue severamente impactado a lo largo de toda la pendiente, debido al posible arrastre del material orgánico y nutrimentos, favorecidos por la erosión hídrica, contribuyendo a la formación de zonas heterogéneas. En consecuencia, el contenido de MO y las poblaciones de BFNVL fueron afectados. Por ello, el potencial de secuestro de carbono fue muy bajo en comparación con otros estudios realizados en cafetales bajo sombra, lo que indica, que bajo estas condiciones, no es apto para competir en el mercado de carbono, afectando negativamente el potencial económicos de la zona. Por todo lo anterior, el desarrollo de estrategias de conservación de suelos fue altamente recomendado para este sitio.

Palabras clave: Sustentabilidad/ pérdida de suelo/ servicios ambientales/ desarrollo rural sustentable/ agroforestería.

\begin{abstract}
In this study was analyzed the carbon sequestration potential of a soil from a shaded coffee plantation, influenced by a slope of $34 \%$. The total carbon sequestered along the slope was quantified by providing leaf litter, branches, mulch and soil. The population of nitrogen fixing bacteria free-living (NFBFL) and the content of organic matter $(\mathrm{OM})$ were used as indicators of soil quality. Finally, the impact of the economic benefits of carbon sequestration under these conditions was determined. The results indicated
\end{abstract}


that the coffee under shade provides favourable conditions for carbon sequestration due to the continuous supply of carbon from leaf litter and branches, with the consequent formation of mulch; however, the total carbon sequestration was severely impacted along the entire slope, due to possible drag of organic material and nutrients, favoured by water erosion, contributing to the formation of heterogeneous areas. Consequently, the content of MO and BFNVL populations were affected. Therefore, the carbon sequestration potential was very low compared with other studies in shaded coffee plantations, indicating that under these conditions, is not eligible to compete in the carbon market, adversely affecting the economic potential of zone. For all these reasons, the development of soil conservation strategies was highly recommended for this site.

Keywords: Sustainability, soil loss, environmental services, sustainable rural development, agroforestry.

\section{INTRODUCCIÓN}

El cambio climático es un tema que desde hace varias décadas ha formado parte de las investigaciones y ha hecho que los gobiernos del mundo incorporen en sus diferentes agendas el pago de servicios ambientales por fijación y almacenamiento de carbono, representando una opción para dar valor agregado a la producción agropecuaria (Ordoñez-Díaz, 1999; DávalosSotelo et al., 2008). Esta estrategia debe ser considerada, diseñada e implementada a corto plazo (Pineda-López et al., 2005), bajo el contexto de pérdida de vegetación debido a los cambios de uso de suelo de bosques a cultivos y pastizales, que son dos de los principales problemas que ponen en peligro al recurso suelo (Torres \& Guevara, 2002; Caballero, 2010). En este sentido, los sistemas agroforestales han sido de gran importancia en algunas regiones tropicales de México (Torquebiau, 2000), debido a que el establecimiento de plantaciones, sistemas agroforestales y la consecuente rehabilitación de bosques degradados sonestrategias a largo plazo (Thuille et al., 2000).

Los bosques son reconocidos como sistemas importantes en servicios ambientales debido a su capacidad de almacenamiento y secuestro de $\mathrm{CO}_{2}$; sin embargo, son los cafetales con sombra los sistemas agroforestales con mayor potencial para ser incorporados en el mercado del carbono (Sartorio, 2002; Pineda, 2005).Estos sistemas cuentan con un sinnúmero de ventajas ecológicas y ambientales, estudios demuestran una contribución importante de éstos como servicios ambientales (Beeret al., 2003). Por un lado, la asimilación de carbono se estima en más del $89 \%$, y ésta corresponde principalmente al carbono del suelo (Ávila et al., 2001), además existe una mejora en la dinámica de nitrógeno y una reducción en la contaminación de mantos freáticos (Ávila et al., 2004). En este sentido, el objetivo de la presente investigación fue analizar el potencial de secuestro de carbono de un suelo proveniente de un cafetal con sombra, influenciado por una pendiente del $34 \%$, mediante la determinación del aporte de carbono de la hojarasca, las ramas y el mantillo, así como la evaluación de indicadores de la calidad del suelo por medio de la estimación de bacterias fijadoras de nitrógeno de vida libre (BFNVL) y el contenido de materia orgánica (MO).

\section{MATERIALES Y MÉTODOS}

El sitio de estudio se localizó en las coordenadas $18^{\circ} 16^{\prime} 20.3^{\prime \prime}$ norte y $94^{\circ} 56^{\prime} 52.5^{\prime \prime}$ oeste, perteneciente al ejido de El Tulín, municipio de Soteapan, al sur de Veracruz. 
Este sitio fue seleccionado, debido a que es representativo de los cafetales marginales, de manejo rústico y típicos de la zona de amortiguamiento de la Reserva de la Biosfera de los Los Tuxtlas (Figura 1A).

El clima es Aw2, con precipitación media anual de 3500 a $4000 \mathrm{~mm}$, temperatura media anual de $24^{\circ} \mathrm{C}$ y con vegetación selva alta perennifolia (INEGI, 2012).
La zona de estudio fue un cafetal con sombra, de 25 años de edad, una pendiente de $34 \%$ y con una longitud de $104 \mathrm{~m}$ de longitud.

La captura de carbono se estudió mediante el muestreo de tres transectos con tres pseudoréplicas, la distancia entre cada uno fue de $30 \mathrm{~m}$.

Cada parcela estudiada fue de $10 \mathrm{~m}^{2}$ dentro del cual, se muestrearon zonas de $0.25 \mathrm{~m}^{2}$ de donde se colectó la hojarasca, el mantillo y el suelo (Figura 1B).

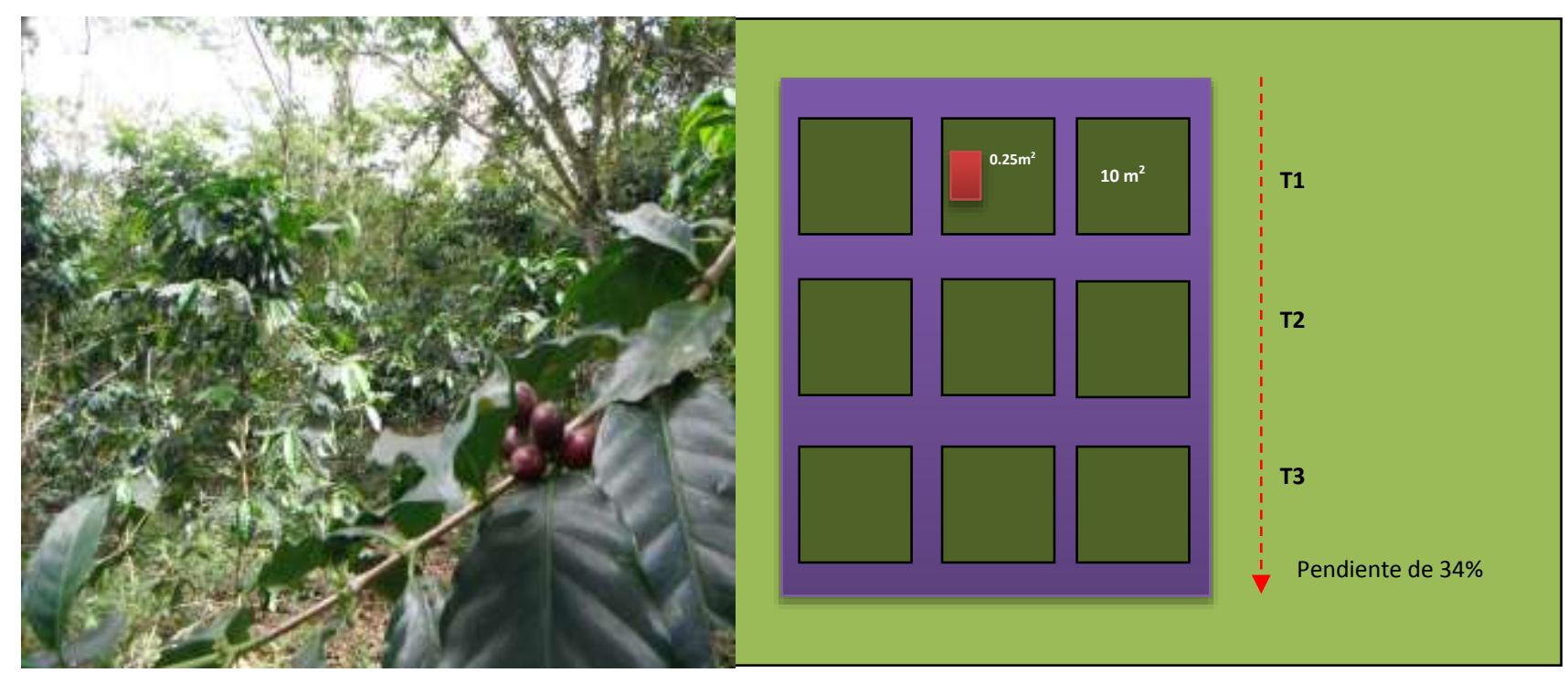

Figura 1. A) Cafetal rústico, típico de la zona y B) Representación esquemática del área de estudio, donde $\mathrm{T} 1$ corresponde a la zona más alta, el T2 a la parte media de la pendiente y el T3 se refiere a la parte más baja de la pendiente.

El aporte del carbono en la hojarasca se ajustó a los lineamientos técnicos descritos para estudios ecológicos e inventarios de biomasa en bosques y sistemas agroforestales (Rendón y Soto, 2007; Rügnitet al., 2009; Espinoza et al., 2012). Para estimar la cantidad de carbono almacenado en las ramas y mantillo se utilizaron las fórmulas propuestas por Dávalos et al. (2008). El carbono orgánico y la materia orgánica del suelo se determinaron de acuerdo a la NOM-021RECNAT-2000 (SEMARNAT, 2003). Se evaluó la población de bacterias fijadoras de nitrógeno como indicador de la calidad del suelo, utilizando el método de cuenta viable por dilución seriada (Madigan et al., 2003) en suelo para las poblaciones de bacterias fijadoras de nitrógeno en vida libre (BFNVL). Se prepararon diluciones decimales seriadas $\left(1 / 10^{5}\right)$ con $10 \mathrm{~g}$ 
de suelo del cafetal en $90 \mathrm{~mL}$ de agua destilada estéril, utilizando el medio de cultivo de carbón combinado (CIAT, 1988). Por último, se determinó el impacto de los beneficios económicos del secuestro de carbono bajo esas condiciones, tomando como referencia el valor utilizado en los proyectos internacionales de 10 $\mathrm{U} \$ \mathrm{t}^{-1}$ (Ávila et al, 2001) y se realizaron las correlaciones entre las variables y ANOVA.

\section{RESULTADOS}

Los resultados indican que la pendiente influyó directamente sobre la acumulación de hojarasca ( yojarasca $\left.=98.253 \mathrm{x}-33.705, \mathrm{R}^{2}=0.999\right)$, ramas $\left(\mathrm{y}_{\text {ramas }}=202.49 \mathrm{x}-103.09, \mathrm{R}^{2}=1\right)$ y la consecuente formación de mantillo (ymantillo $=$ $\left.320.85 x-228.48, R^{2}=0.9424\right)$, lo que podría deberse al arrastre de las partículas hacia la parte baja de la pendiente (Figura 2A). En este sentido, el pago estimado por secuestro de carbono es mayor en suelo y en forma acumulativa en la zona más baja de la pendiente (Figura 2B). El porcentaje de carbono en el suelo y su contenido de materia orgánica no se vio afectado en calidad cantidad por gramo de suelo seco analizado, manteniéndose valores promedios entre $2.5 \mathrm{y}$ $3 \%$ para $\mathrm{MO}$ y el $\mathrm{CO}$ osciló entre 1.5 a 1.8 . (Figura 3A); sin embargo, si fueron disminuidas las BFNVL, siendo superiores en la parte media que en la parte alta y baja (Figura 3B), esta última posiblemente al exceso de humedad y la mayor mineralización de carbono.

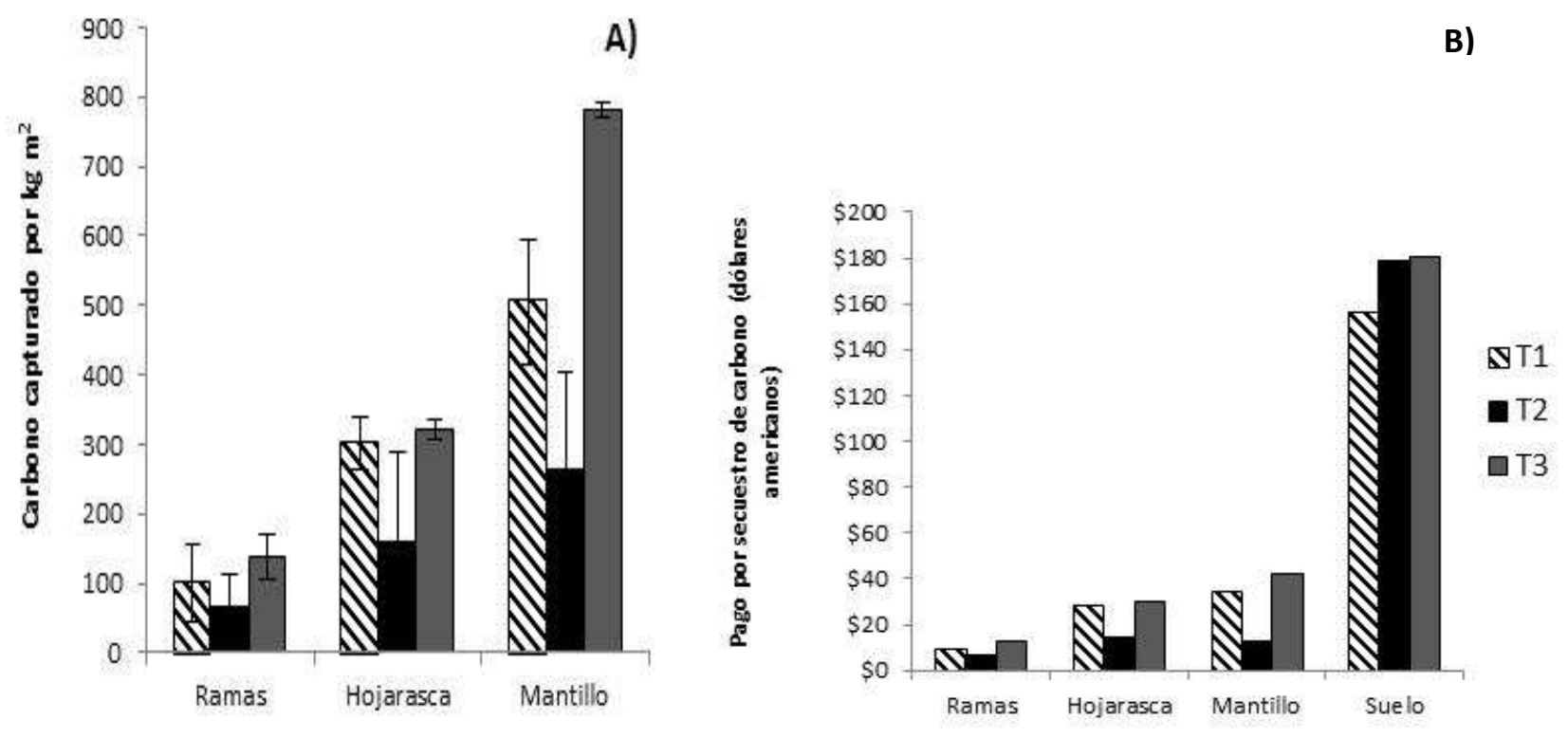

Figura 2. A) Aporte de carbono por parte de las ramas, la hojarasca y el mantillo por metro cuadrado y B) estimación de pago, en dólares americanos, por concepto de secuestro de carbono t ha ${ }^{-1}$ a lo largo de la pendiente; donde T1 es la parte más alta de la pendiente, T2 la media y T3 la baja. 

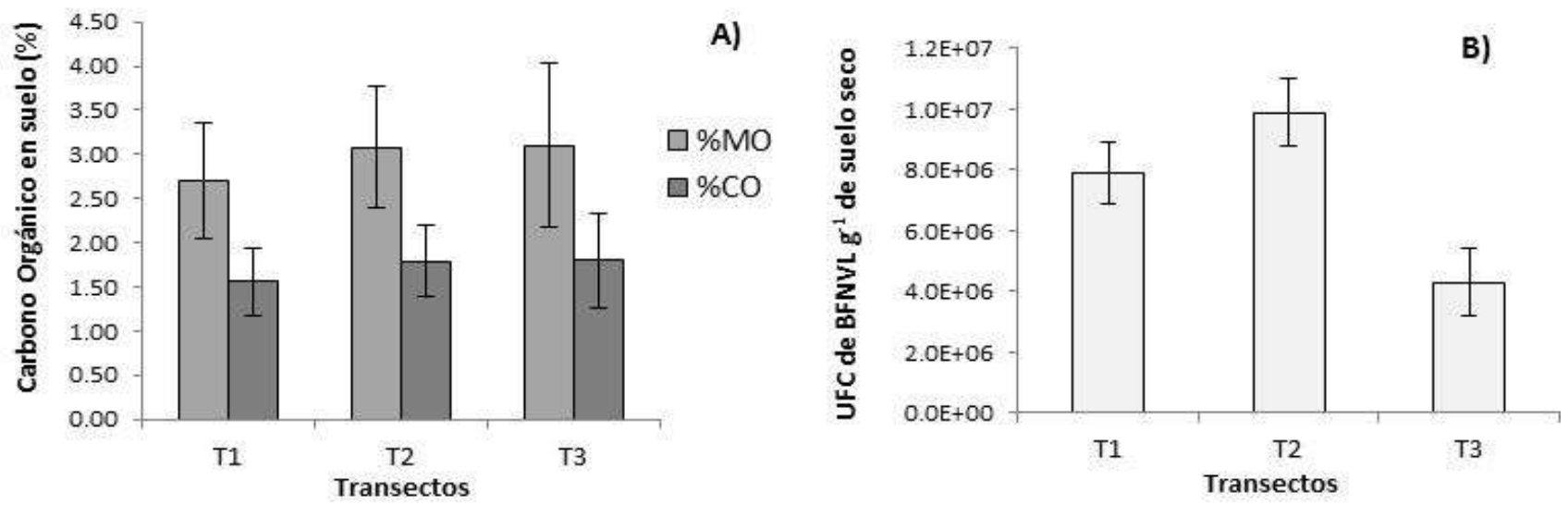

Figura 3. A) Carbono orgánico (\%) en el suelo; y B) Unidades Formadoras de colonias (UFC) de Bacterias Fijadoras de Nitrógeno de Vida Libre (BFNVL) por gramo de suelo seco a lo largo de la pendiente; donde T1 es la parte más alta, T2 (media) y T3 (baja).

\section{DISCUSIÓN}

Los sistemas agroforestales son de vital importancia en el estado de Veracruz (BautistaTolentino et al., 2011) caracterizados por su efecto en suelos (Burgarín et al., 2010), por sus funciones ecológicas (Giraldo et al., 2008), por su rentabilidad (Zamudio et al., 2010) o por ser una alternativa en el pago de servicios ambientales (Pérez-García et al., 2009). Sin embargo, dada su complejidad y diversidad de ambientes, los resultados aquí presentados concuerdan en que la hojarasca es la principal fuente de carbono para la síntesis enzimática del humus, debido a que está compuesta de una fracción lábil que se descompone con rapidez (Chavez-Vergara et al., 2014), en consecuencia el mantillo está determinados por el balance entre la caída de hojarasca y su descomposición mediante factores químicos, físicos y biológicos; por lo que éste es el principal sitio de almacenamiento de carbono y de elementos minerales en el suelo de algunos ecosistemas (Martínez y Sarukhan, 1993; Li et al., 2014)). Es también un componente muy dinámico y fundamental en los procesos de flujo de energía y circulación de nutrientes dentro de ecosistema (Falkowski et al., 2000).

Si bien el contenido de materia orgánica en este sitio es alto, la pérdida de suelo es de hasta $6 \mathrm{~cm}$ por año (aproximadamente 570 ton $\mathrm{ha}^{-1} \mathrm{año}^{-1}$ ), además el contenido de nitrógeno en el suelo se estima en $0.36 \%$ en promedio (muy rico), este se pierde por lixiviación o por erosión hídrica, afectando a las poblaciones de BFNVL (Hernández et al., 2013). Aunque existen estudios que enumeran las ventajas de estos sistemas desde el punto vista ecológico, ambiental, social y económico, las condiciones de alta pendiente pueden ocasionar el arrastre de materia orgánica de la parte alta, mientras que la zona media queda parcialmente desprotegida en comparación con la parte alta y la parte baja, indicando que en la parte baja es donde existe una mayor acumulación de mantillo (García et $a l .$, 2006), mismo que se pierde en el corto plazo por efecto del arrastre por escurrimiento superficial y teniendo efectos significativos sobre el secuestro de carbono debidos a pendiente, altitud y características edáficas (Hernández-Vásquez, 2012). Esto pone en peligro la competitividad socioeconómica de 
estos cafetales con respecto al pago por servicios ambientales de estos agroecosistemas (Klooster \& Masera, 2000; Ninan y Inoue, 2013) y las bacterias fijadoras de nitrógeno de vida libre (Mantilla-Paredes et al., 2009; Levy-Booth et al., 2014).

\section{CONCLUSIONES}

El potencial de secuestro de carbono de un suelo proveniente de un cafetal con sombra, influenciado por una pendiente del $34 \%$ fue severamente afectado, debido a que el aporte de carbono de la hojarasca, las ramas y el mantillo fue mayor en la parte baja de la pendiente; sin embargo, esto no afecta el contenido de carbono y materia orgánica por gramo de suelo seco; pero si su cantidad y la cantidad de BFNVL, siendo ésta menor en la zonas baja y alta.

\section{AGRADECIMIENTOS}

Este estudio formó parte del proyecto "Diagnóstico para el manejo de la microcuenca de Michapan, Veracruz", que contó con financiamiento por el Programa de Mejoramiento del Profesorado (PROMEP) de la SEP, a través de la convocatoria Fortalecimiento a Cuerpos Académicos. Se agradece además el apoyo a productores del ejido El Tulín, municipio de Soteapan, por las facilidades prestadas para el trabajo de campo.

\section{LITERATURA CITADA}

Ávila, G.; F. Jiménez; J. Beer; M. Gómez y M. Ibrahim. 2001. Almacenamiento, fijación de carbono y valoración de servicios ambientales en sistemas agroforestales en Costa Rica. Agroforestería de las Américas, 8(30): 32-35.

Ávila, H.;J. M. Harmandy Dambrine, E. 2004. Dinámica del nitrógeno en el sistema agroforestal Coffeaarabica con
Eucalyptusdeglupta en la Zona Sur de Costa Rica. Agroforestería de las Américas, 41-42: 83-91.

Bautista-Tolentino, M.; S. López-Ortíz; P. Pérez-Hernández; M. Vargas-Mendoza; F.Gallardo-López y F. C. GómezMerino. 2011. Sistemas agro y silvopastoril en la comunidad El Limón, municipio de Paso de ovejas, Veracruz, México. Tropical and Subtropical Agroecosystems, 14: 63-76.

Beer, J.;C. Harvey; M. Ibrahim; J. M. Harmand; E. Somarriba y F. Jiménez. 2003. Servicios ambientales de los sistemas agroforestales. Agroforestería de las Américas, 10(37): 80-87.

Bugarín, J.;J. L. Bojórquez; C. Lemus; R. M. Murray; A. Hernández; H. Ontiveros y J. Aguirre. 2010. Comportamiento de algunas propiedades físicoquímicas del suelo con diferente sistema silvopastoril en la llanura norte de Nayarit. Cultivos Tropicales, 31(2): 48-55.

Caballero, M. D. 2010. La verdadera cosecha maderable en México. Rev. Mex. Cien. For. 1:1-16.

Chavez-Vergara, B.; A., Merino; G. VázquezMarrufo y F. García-Oliva. 2014. Organic matter dynamics and microbial activity during decomposition of forest floor under two native neotropical oak species in a temperate deciduous forest in Mexico. Geoderma, 235-236, 133-145. https://doi.org/10.1016/j.geoderma.2014.

CIAT (Centro Internacional de Agricultura Tropical). 1988. Simbiosis leguminosarizobio. Manual de métodos de evaluación, selección y manejo agronómico. Ed. Rev. Proyecto CIATUNDP de evaluación, selección y manejo de la simbiosis leguminosarizobio para aumentar la fijación de nitrógeno. Sección de Microbiología de 
Suelos en el Programa de Pastos Tropicales y Sección de Microbiología del Programa de Fríjol (comps.) Cali, Colombia. 178 p. Dávalos-Sotelo,R., M. M. I. Rodrigues y Pinillos-Cueto, $\quad$ E. 2008. Almacenamiento de Carbono. In: R. Manson; V. Hernández-Ortíz; S. Gallina y K. Mehltreter (EDS).Agrosistemas cafetaleros en Veracruz: Biodiversidad, Manejo y Conservación México: INE. Espinoza-Dominguez, W.;L. Krishnamurthy yA. Y. R. Vázquez-Alarcon. 2012. Almacén de carbono en sistemas agroforestales con café. Rev. Chapingo, 57-70.

https://doi.org/10.5154/r.rchscfa.2011.04 Falkowski, P.; R. J. Scholes; E. Boyle, J. Canadell; D. Canfield y J.Elser2000. The global carbon cycle: a test of our knowledge of Earth as a system. Science, 290: 291-296.

https://doi.org/10.1126/science.290.5490

García, O. F.; G. Hernández yL. J. F. Gallado.2006 Comparison of Ecosystem $\mathrm{C}$ pools in three forest in Spain and Latin America. Ann. For. Sci., 63: 519-523. https://doi.org/10.1051/forest:2006034

Giraldo,A.;M. Zapata y E. Montoya. 2008. Captura y flujo de carbono en un sistema silvopastoril de la zona Andina Colombiana. Asociación Latinoamericana de Producción Animal, 14(4): 241-245.

Hernández, R. A. H.; F. A. F. A. Gómez.; A. Retureta; D. Vázquez-Luna;O. N. Rodríguez; M. C. Cuevas-Díaz; R. D. I., Torres y A. K. C. Lozano. 2013. Diagnóstico para el manejo de la microcuenca de Michapan, Veracruz. Componente Suelo y Agua. Informe técnico del proyecto. Universidad Veracruzana.

Hernández-Vasquez, E.;G. Campos Ángeles;J. Enríquez-Del Valle, G. Rodríguez-Ortíz
Vázquez-Luna et al., 2018

y V. Velasco-Velasco. Captura de carbono por Jinicuil schltdl en un sistema agroforestal de café bajo sombra. Rev. Mex. Cien., 3(9), 11.

https://doi.org/10.29298/rmcf.v3i9.536

INEGI. 2012. Guía para la interpretación cartográfica. Uso de suelo y vegatación. Escala 1: 250 000. Serie IV. Instituto Nacional de Estadística y Geografía, INEGI.México. 129 p.

Klooster, D. y O. Masera. 2000. Community forest management in Mexico: carbon mitigation and biodiversity conservation through rural development. Global Environmental Change, 10(4), 259-272. https://doi.org/10.1016/S0959-3780(00)0 Levy-Booth, D. J.; C. E., Prescott, y S. J. Grayston. 2014. Microbial functional genes involved in nitrogen fixation, nitrification and denitrification in forest ecosystems. Soil Biology and Biochemistry, https://doi.org/10.1016/j.s Li, C.;J. Moore-Kucera; J. Lee; A. Corbin;M. Brodhagen;C. Miles, y D. Inglis. 2014. Effects of biodegradable mulch on soil quality. Applied Soil Ecology, 79, 59-69. https://doi.org/10.1016/j.apsoil.2014.02.012 Madigan., M.T.;Martinko, J.M. y J. Parker. 2000. Brock. Biología de los microorganismos. In:Gacto FM; García I.; González V. A. T.; Guerrero M. V.; Sánchez O. M. (EDS). 9a Ed. Prentice Hall Iberia. Madrid, España. 1064 p.

Mantilla-Paredes, A.J.; G.I. Cardona; C.P. PeñaVenegas; U. Murcia; M. Rodríguez y M.M. Zambrano. 2009. Distribución de bacterias potencialmente fijadoras de nitrógeno y su relación con parámetros físicoquímicos en suelos con tres coberturas vegetales en el sur de la Amazonia colombiana. Rev. Biol. Trop. 57: 915-927. https://doi.org/10.15517/rb Martínez, Y., A. yJ. Sarukhan. 1993. Cambios estacionales del mantillo en el suelo de un bosque tropical caducifolio y uno 
subcaducifolio en Chamela, Jalisco, México.

Acta Botánica Mexicana, 21, 1-6.

https://doi.org/10.21829/abm21.1993.662

Ninan, K.y M. Inoue. 2013. Valuing forest ecosystem services: What we know and what we don't. Ecological Economics., 93, 137-149. https://doi.org/10.1016/j.ec

Ordoñez-Díaz, J.A.B. 1999. Estimación de la captura de carbono en un estudio de caso. Primera edición, Instituto Nacional de Ecología-SEMARNAP. 73 p.

Pérez-García, N.;M. Rueda-González;G. E. Rojo-Martínez;R. Martínez- Ruíz;B. Ramírez-Valverde; y J. P. JuárezSánchez. 2009. El bambú (Bambusaspp.) como sistema agroforestal: una alternativa de desarrollo mediante el pago por servicios ambientales en la sierra nororiental del Estado de Puebla. Ra Ximhai, 5(3): 335-346. https://doi.org/10.35197/rx.05.03.2009.0 Pineda, L. M. D. R.;G. Ortiz Ceballos yL. R. Sánchez Velásquez. 2005. Los cafetales y su papel en la captura de carbono: un servicio ambiental aún no valorado en Veracruz. Madera y Bosques, 11(2) 3-14. https://doi.org/10.21829/myb.2005.1121253 Pineda-López, M. R. 2005. Los cafetales y su captura de carbono: un servicio ambiental aún no valorado en

Veracruz.Madera y Bosques 11(2): 3-14.

https://doi.org/10.21829/myb.2005.1121253

Rendón C., N. yL. SotoP. 2007. Metodología rápida para la estimación y monitoreo de captura de carbono. ECOSUR. Chiapas, México. 47 p.

Rügnitz,M. T.; L. M. ChacónyR. Porro. 2009. Guía para la determinación de carbono en pequeñas propiedades rurales.Lima,
Perú. Centro Mundial de Agroforestería, Consorcio Iniciativa Amazónica.92 p.

Sartorio, B. Á. 2002. Los cafetales de sombra como proveedores de servicios ambientales. Ciencia y Mar, 17-18.

SEMARNAT. 2003. Norma Oficial Mexicana NOM-021-SEMARNAT-2000, que establece las especificaciones de fertilidad y clasificación de suelos, estudio, muestreo y análisis. Secretaría del Medio Ambiente y Recursos Naturales. Diario Oficial de la Federación, 23/04/2003. México, DF, México. 85 pp.

Thuille, A. N. Buchmanny E. D. Schulze. 2000. Carbon stocks and soil respiration rates during deforestation, grassland use and subsequent Norway spruce afforestation in the Southern Alps, Italy. Tree Physiology, 20, 849-857.

https://doi.org/10.1093/treephys/20.13.84 Torquebiau, E.F. 2000. A renewed perspective on agroforestry concepts and classification.Life Sciences, 323: 10091017. https://doi.org/10.1016/S0764-446

Torres T. J. M. yS. A. Guevara 2002. El potencial de México para la producción de servicios ambientales: captura de carbono y desempeño hidráulico. Gaceta Ecológica INE- SEMARNAT No. 63.

Zamudio S. F. J.;J. L. Romo Lozano y J. O. A. Cervantes Carrillo. 2010. Evaluación financiera y de riesgo de una plantación forestal comercial en Zihuateutla, Puebla. Revista Chapingo Serie Ciencias Forestales y del Ambiente, 16(1): 69-78. 
Copyright (c) 2018 Dinora Vázquez Luna, Maria del Carm en Cuevas Diaz, Teresita de Jesús Perera Escamilla,

Ángel Héctor Hernández Rom eroy Alejandro Retureta Aponte

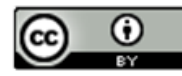

Este tex to está protegido por una licencia licencia Creative Commons 4.0.

Usted es libre para Compartir —copiar y redistribuir el material en cualquier medio o form ato- y Adaptar el documento - remezclar, transformar y crear a partir del material- para cualquier propósito, incluso para fines comerciales, siempre que cumpla la condición de:

Atribución: Usted debe dar crédito a la obra original de manera adecuada, proporcionar un enlace a la licencia, e in dicar si se han realizado cam bios. Puede hacerlo en cualquier form a razonable, pero no de form a tal que sugiera que tiene el apoyo del licenciante olo recibe por el uso que hace de la obra.

Resumencielicencia - Textocompletodelalicencia 